\title{
Секвенциальная аксиоматизация паранормальной логики PContPComp ${ }^{1}$
}

\author{
В. М. Попов
}

ABstract. The sequent calculas axiomatizing paranormal logic PContPComp
is presented.
Ключевые слова: секвенция, исчисление, паранормальная логика,
паранепротиворечивая логика, параполная логика

Предлагается удобное для осуществления поиска доказательства секвенциальное исчисление, аксиоматизирующее логику PContPComp из [3].

Язык $L$ логики PContPComp есть стандартный пропозициональный язык с алфавитом $\{\&, \vee, \supset, \neg),,\left(, p_{1}, p_{2}, p_{3}, \ldots\right\}$ (разумеется, символы \&, $\vee$ и $\supset$ - бинарные, а символ $\neg-$ унарная логические связки языка $L$, ) и ( - технические символы языка $L$, символы $p_{1}, p_{2}, p_{3}, \ldots$ - пропозициональные переменные этого языка). Принимаем обычные соглашения об опускании скобок в $L$-формулах и используем «формула» вместо «L-формула». Логикой называем непустое множество формул, замкнутое относительно правила подстановки в $L$ и правила modus ponens в $L$. Теорией логики $\mathbf{L}$ называем множество формул, включающее логику $\mathbf{L}$ и замкнутое относительно правила modus ponens в $\mathbf{L}$. Множество всех формул называем тривиальной теорией. Противоречивой теорией логики $\mathbf{L}$ называем такую теорию $\mathbf{T}$ логики $\mathbf{L}$, что для некоторой формулы $A$ верно следующее: $A \in \mathbf{T}$ и $\neg A \in \mathbf{T}$. Паранепротиворечивой теорией логики $\mathbf{L}$ называем такую противоречивую теорию $\mathbf{T}$ логики $\mathbf{L}$, что $\mathbf{T}$ не есть тривиальная теория. Паранепротиворечивой логикой называем такую логику $\mathbf{L}$, что существует паранепротиворечивая теория логики

\footnotetext{
${ }^{1}$ Работа выполнена при поддержке РГНФ, проект № 10-03-00570а.
} 
$\mathbf{L}$. Полной теорией логики $\mathbf{L}$ называем такую теорию $\mathbf{T}$ логики $\mathbf{L}$, что для всякой формулы $A$ верно: $A \in \mathbf{T}$ или $\neg A \in \mathbf{T}$. Параполной теорией логики $\mathbf{L}$ называем такую теорию $\mathbf{T}$ логики $\mathbf{L}$, что $\mathbf{T}$ не является полной теорией логики $\mathbf{L}$ и всякая полная теория логики $\mathbf{L}$, включающая $\mathbf{T}$, есть тривиальная теория. Параполной логикой называем такую логику $\mathbf{L}$, что существует параполная теория логики L. Паранормальной логикой называем логику, которая является паранепротиворечивой и параполной логикой. Следуя [3], строим исчисление HPContPComp гильбертовского типа и определяем логику PContPComp. Язык исчисления HPContPComp есть $L$. Правило modus ponens в $L$ является единственным правилом вывода этого исчисления. Выводы (в частности, доказательства) в HPContPComp строятся обычным для гильбертовского типа исчислений образом (см. [1], [4]). Множеству всех аксиом исчисления HPContPComp принадлежат все те и только те формулы, каждая из которых имеет хотя бы один из следующих видов (здесь и далее $A, B$ и $C$ есть формулы):

(I) $(A \supset B) \supset((B \supset C) \supset(A \supset C))$,

(II) $A \supset(A \vee B)$,

(III) $A \supset(B \vee A)$,

(IV) $(A \supset C) \supset((B \supset C) \supset((A \vee B) \supset C))$,

(V) $(A \& B) \supset A$,

(VI) $(A \& B) \supset B$

(VII) $(C \supset A) \supset((C \supset B) \supset(C \supset(A \& B)))$,

(VIII) $(A \supset(B \supset C)) \supset((A \& B) \supset C)$,

(IX) $((A \& B) \supset C) \supset(A \supset(B \supset C))$,

(X) $((A \supset B) \supset A) \supset A$,

(XI) $\neg(A \vee B) \supset(\neg A \& \neg B)$,

(XII) $(\neg A \& \neg B) \supset \neg(A \vee B)$,

(XIII) $\neg(A \& B) \supset(\neg A \vee \neg B)$,

$(\mathrm{XIV})(\neg A \vee \neg B) \supset \neg(A \& B)$,

$(\mathrm{XV}) \neg(A \supset B) \supset(\neg A \& B)$,

(XVI) $(\neg A \& B) \supset \neg(A \supset B)$,

(XVII) $\neg \neg A \supset A$,

(XVIII) $A \supset \neg \neg A$, 
$(\mathrm{XIX})(A \& \neg A) \supset(B \vee \neg B)$.

Множество всех формул, доказуемых в HPContPComp, обозначаем через PContPComp. Согласно [3], PContPComp является паранормальной логикой. Эта логика есть пересечение паранепротиворечивой (но не параполной) логики PCont и параполной (но не паранепротиворечивой) логики РComp (см. [2], [3]). Построим секвенциальное исчисление GPContPComp. Алфавит А языка этого секвенциального исчисления есть объединение алфавита языка $L$ с двухэлементным множеством $\{\boldsymbol{g}, \rightarrow\}$ символов. Непустой последовательностью формул называем слово в алфавите $\mathbf{A}$, имеющее вид $A_{1}, \ldots, A_{n}$, где $n$ есть целое положительное число, а $A_{1}, \ldots, A_{n}$ есть формулы. В случае, когда $n=1$, $A_{1}, \ldots, A_{n}$ есть $A_{1}$. Пустой последовательностью формул называем пустое слово (в алфавите $\mathbf{A}$ ). Называем $\pi$ последовательностью формул, если $\pi$ есть непустая последовательность формул или пустая последовательность формул. Условимся обозначать через $\Gamma, \Sigma, \Delta$ и $\Theta$ последовательности формул. Секвенцией называем слово в алфавите $\mathbf{A}$, имеющее вид $\Gamma \rightarrow \Delta$.

Пусть $S e q$ есть множество всех секвенций и для всякого целого положительного числа $n S e q^{n}$ есть $n$-ная декартова степень множества $S e q$. Называем $R$ секвенциальным правилом, если для некоторого целого положительного числа $n R \subseteq S e q^{n+1}$. Называем П применением секвенциального правила $R$, если $\Pi \in R$. Для описания применений секвенциальных правил потребуется операция •. Можно доказать, что существует единственная бинарная операция (обозначаем ее через •) на множестве всех последовательностей формул, удовлетворяющая следующим трем условиям (а), (б) и (в).

(a) $\Gamma \bullet \Delta=\Gamma$, если $\Delta$ есть пустая последовательность формул.

(б) $\Gamma \bullet \Delta=\Delta$, если $\Gamma$ есть пустая последовательность формул.

(в) $\Gamma \bullet \Delta=\Gamma, \Delta$, если ни $\Gamma$, ни $\Delta$ не есть пустая последовательность формул.

Правилами исчисления GPContPComp являются определяемые ниже двадцать пять секвенциальных правил (и только они). 
Каждое из этих секвенциальных правил определяем путем указания общего вида применения данного правила. При этом, следуя традиции, пользуемся двухмерным представлением упорядоченных пар секвенций и упорядоченных троек секвенций.

$$
\begin{aligned}
& \frac{((\Gamma \bullet A, B) \bullet \Delta) \rightarrow \Theta}{(\Gamma \bullet B, A) \bullet \Delta) \rightarrow \Theta} \quad(\Pi Л), \quad \frac{\Gamma \rightarrow((\Delta \bullet A, B) \bullet \Theta)}{\Gamma \rightarrow((\Delta \bullet B, A) \bullet \Theta)}(\Pi \Pi), \\
& \frac{(A, A \bullet \Gamma) \rightarrow \Theta}{(A \bullet \Gamma) \rightarrow \Theta}(\text { СЛ }), \quad \frac{\Gamma \rightarrow(\Theta \bullet A, A)}{\Gamma \rightarrow(\Theta \bullet A)} \quad(\mathrm{C \Pi}), \\
& \frac{\Gamma \rightarrow \Theta}{(A \bullet \Gamma) \rightarrow \Theta} \text { (ДЛ), } \quad \frac{\Gamma \rightarrow \Theta}{\Gamma \rightarrow(\Theta \bullet A)}(\text { ДП }), \\
& \frac{(A \bullet \Gamma) \rightarrow \Theta}{((A \& B) \bullet \Gamma) \rightarrow \Theta}\left(\mathrm{BK}_{1}\right), \quad \frac{(A \bullet \Gamma) \rightarrow \Theta}{((B \& A) \bullet \Gamma) \rightarrow \Theta}\left(\mathrm{BK}_{2}\right), \\
& \frac{\Gamma \rightarrow(\Theta \bullet A) \Gamma \rightarrow(\Theta \bullet B)}{\Gamma \rightarrow(\Theta \bullet(A \& B))}(\mathrm{BK} \Pi) \\
& \frac{((\neg A) \bullet \Gamma) \rightarrow \Theta \quad((\neg A) \bullet \Gamma) \rightarrow \Theta}{((\neg(A \& B)) \bullet \Gamma) \rightarrow \Theta}(\text { ВOKЛ), } \\
& \frac{\Gamma \rightarrow(\Theta \bullet(\neg A))}{\Gamma \rightarrow(\Theta \bullet \neg(A \& B))}\left(\text { BOПK }_{1}\right), \quad \frac{\Gamma \rightarrow(\Theta \bullet(\neg A))}{\Gamma \rightarrow(\Theta \bullet \neg(B \& A))}\left(\text { BOПK }_{2}\right),
\end{aligned}
$$

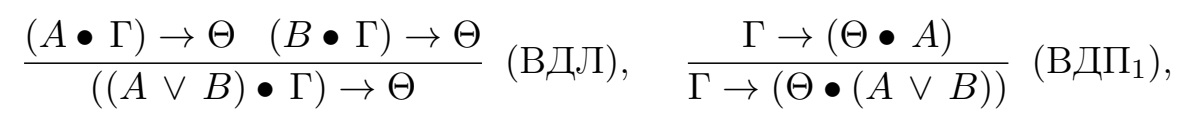

$$
\begin{aligned}
& \left.\frac{\Gamma \rightarrow(\Theta \bullet A)}{\Gamma \rightarrow(\Theta \bullet(B \vee A))}(\text { ВДП }), \quad \frac{((\neg A) \bullet \Gamma) \rightarrow \Theta}{((\neg(A \vee B)) \bullet \Gamma) \rightarrow \Theta} \text { (ВОДЛ }{ }_{1}\right),
\end{aligned}
$$

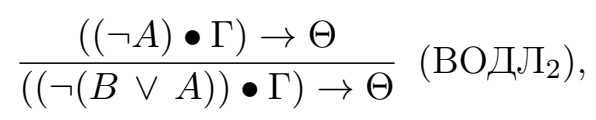




$$
\begin{aligned}
& \frac{\Gamma \rightarrow(\Theta \bullet(\neg A)) \quad \Gamma \rightarrow(\Theta \bullet(\neg B))}{\Gamma \rightarrow(\Theta \bullet(\neg(A \vee B)))} \text { (ВОДП), } \\
& \left.\frac{\Gamma \rightarrow(\Theta \bullet A)(B \bullet \Sigma) \rightarrow \Theta}{(((A \supset B) \bullet \Gamma) \bullet \Sigma) \rightarrow(\Theta \bullet \Sigma)} \quad \text { ВИЛ }\right), \frac{(A \bullet \Gamma) \rightarrow \Theta}{\Gamma \rightarrow(\Theta \bullet(A \supset B)))} \text { (ВИП), } \\
& \left.\frac{(A, \neg B \bullet \Gamma) \rightarrow \Theta}{((\neg(B \supset A)) \bullet \Gamma) \rightarrow \Theta} \text { (ВОИЛ }\right) \\
& \frac{\Gamma \rightarrow(\Theta \bullet A)) \quad \Gamma \rightarrow(\Theta \bullet(\neg A))}{\Gamma \rightarrow(\Theta \bullet(\neg(A \supset B)))}(\text { ВОИП }) \\
& \frac{(A \bullet \Gamma) \rightarrow \Theta}{(\neg \neg A \bullet \Gamma) \rightarrow \Theta} \text { (ВООЛ), } \quad \frac{\Gamma \rightarrow(\Theta \bullet A)}{\Gamma \rightarrow(\Theta \bullet \neg \neg A)} \text { (ВООП), } \\
& \frac{\Gamma \rightarrow(\Theta \bullet A)(B \bullet \Sigma) \rightarrow \Theta}{(((A \supset B) \bullet \Gamma) \bullet \Sigma) \rightarrow(\Theta \bullet \Sigma)} \text { (сечение) }
\end{aligned}
$$

Множество всех основных секвенций исчисления GPContPComp есть множество всех секвенций, каждая из которых имеет вид $A \rightarrow A$ или $A, \neg A \rightarrow B, \neg B$. Выводы в секвенциальном исчислении GPContPComp строятся обычным для секвенциального типа исчислений образом (см., например, [1] и [4]). Для GPContPComp имеет место теорема об устранимости сечения (подробное доказательство этой теоремы методом Генцена, предложенным в [1], проведено в дипломной работе Знаменской Н.А. «Теорема об устранимости сечения для секвенциального исчисления, аксиоматизирующего пересечение паранепротиворечивой логики Розоноэра с ее параполным напарником» (МГУ, философский факультет, кафедра логики, 2010, научный руководитель Попов В.М.)).

Тот факт, что секвенциальное исчисление GPContPComp аксиоматизирует логику PContPComp, устанавливает нижеследующая теорема.

TЕОРЕМА. Для всякой формуль А: секвениия $\rightarrow$ А доказуема в GPContPCoтр тогда и только тогда, когда A $\in$ PContPComp. 


\section{Литература}

[1] Генцен Г. Исследования логических выводов // Математическая теория логического вывода. М., 1967.

[2] Знаменская Н.A., Попов В.M. Паранормальная логика PContPComp как пересечение паранепротиворечивой логики PCont и параполной логики PComp // Шестые смирновские чтения по логике. Материалы международной научной конференции 17-19 июня 2009. М., 2009. С. 63-65.

[3] Попов B.M. Между Раг и множеством всех формул// Шестые смирновские чтения по логике. Материалы международной научной конференции 17-19 июня 2009. М., 2009. С. 93-95.

[4] Смирнов В.А. Формальный вывод и логические исчисления//Смирнов В.А. Теория логического вывода. М., 1999. С. 16-233. 